\title{
Tratamento de fratura nasal por redução fechada: relato de caso
}

\author{
Treatment of nasal fracture by closed reduction: case report \\ Tratamiento de la fractura nasal por reducción cerrada: \\ reporte de un caso
}

Raquel Barroso Parra da SILVA ${ }^{1}$

Gabriel Mulinari dos SANTOS ${ }^{1}$

Luara Teixeira COLOMBO ${ }^{1}$

Tárik Ocon Braga POLO ${ }^{2}$

Leonardo Perez FAVERANI ${ }^{3}$

Idelmo Rangel GARCIA JÚNIOR ${ }^{3}$

${ }^{1}$ Mestrando em Cirurgia e Traumatologia Bucomaxilofacial, Faculdade de Odontologia de Araçatuba UNESP-Univ. Estadual Paulista, 16015-050 Araçatuba-SP, Brasil

${ }^{2}$ Doutorando em Cirurgia e Traumatologia Bucomaxilofacial, Faculdade de Odontologia de Araçatuba, UNESP-Univ. Estadual Paulista, 16015-050 Araçatuba-SP, Brasil

${ }^{3}$ Professor Cirurgia e Traumatologia Bucomaxilofacial, Faculdade de Odontologia de Araçatuba, UNESP-Univ. Estadual Paulista, 16015-050 Araçatuba-SP, Brasil

\section{Resumo}

As fraturas nasais são as fraturas mais frequentes em face, devido à proeminência dos ossos próprios do nariz (OPN) em relação ao restante da face, sendo neste o primeiro impacto frente a um trauma. As fraturas nasais (FN) podem ser tratadas cirurgicamente ou não, considerando-se as condições estética e funcional do paciente. O presente trabalho tem como objetivo relatar um caso clínico de um paciente de 38 anos, sexo masculino, melanoderma, vítima de agressão física, relatando dificuldade respiratória e queixas álgicas em face. Ao exame clínico notou-se rinoescoliose significativa em lado direito e impermeabilidade das vias aéreas. $\mathrm{O}$ exame de imagem por tomografia computadorizada revelou fratura OPN com fratura e desvio de septo nasal. Levando-se em conta estes resultados, propôs-se tratamento cirúrgico sob anestesia geral, a qual foi realizada com reposição dos fragmentos ósseos, seguida de tamponamento. Para sustentação e manutenção do contorno nasal, realizou-se curativo em gesso para a manutenção do contorno nasal. No pós-operatório foi possível notar resultado satisfatório funcional, com patência das vias aéreas, e também estético, retomando aspecto bastante semelhante às características originais prévias ao trauma.

Descritores: Fraturas Ósseas; Fixação de Fratura; Traumatismos Faciais.

\begin{abstract}
Nasal fractures are the most frequent fractures on the face, due to the prominence of the own bones of the nose (OPN) in relation to the rest of the face, being in this the first impact against a trauma. Nasal fractures (FN) can be treated surgically or not, considering the aesthetic and functional conditions of the patient. The present study aims to report a clinical case of a 38 - year - old male patient, melanoderma, a victim of physical aggression, reporting respiratory distress and pain complaints in the face. Clinical examination revealed significant rhinoscholosis on the right side and airway impermeability. Computed tomography imaging revealed OPN fracture with fracture and nasal septum deviation. Taking these results into account, surgical treatment was proposed under general anesthesia, which was performed with replacement of the bone fragments, followed by tamponade. To support and maintain the nasal contour, gypsum dressing was performed to maintain the nasal contour. In the postoperative period, it was possible to notice a satisfactory functional result, with patency of the airways, and also aesthetic, resuming an aspect quite similar to the original characteristics prior to the trauma.
\end{abstract}

Descriptors: Fractures, Bone; Fracture Fixation; Facial Injuries

\section{Resumen}

Las fracturas nasales son las fracturas más frecuentes en la cara, debido a la prominencia de los huesos propios de la nariz $(\mathrm{OPN})$ en relación al resto de la cara, siendo este el primer impacto frente a un trauma. Las fracturas nasales (FN) pueden ser tratadas quirúrgicamente o no, considerando las condiciones estética y funcional del paciente. El presente trabajo tiene como objetivo relatar un caso clínico de un paciente de 38 años, sexo masculino, melanoderma, víctima de agresión física, relatando dificultad respiratoria y quejas álgicas en cara. En el examen clínico se observó rinoescoliosis significativa en el lado derecho e impermeabilidad de las vías aéreas. El examen de imagen por tomografía computarizada reveló fractura OPN con fractura y desvío de septo nasal. Teniendo en cuenta estos resultados, se propuso tratamiento quirúrgico bajo anestesia general, la cual fue realizada con reposición de los fragmentos óseos, seguida de taponamiento. Para la sustentación y mantenimiento del contorno nasal, se realizó curativo en yeso para el mantenimiento del contorno nasal. En el postoperatorio fue posible notar un resultado satisfactorio funcional, con patencia de las vías aéreas, y también estético, retomando un aspecto bastante similar a las características originales previas al trauma.

Descriptores: Fracturas Óseas; Fijación de Fractura; Traumatismos Faciales.

\section{INTRODUÇÃO}

Devido à sua anatomia de projeção triangular e sua estrutura delgada, o nariz é dentre os ossos da face o mais atingido durante os traumas, representando $49 \%$ das fraturas maxilo-facias ${ }^{1-3}$. A composição anatômica nasal se dá basicamente pelos ossos próprios do nariz, sendo o restante de sua constituição formada por junções do osso frontal, etmoide, esfenoide e maxilar superior. Há ainda a presença de estruturas cartilaginosas, sendo a porção anterior constituída pelas cartilagens alares, o dorso pela cartilagem lateral e a região interna pelo septo cartilaginoso que se apoia no osso vômer ${ }^{4}$.

O diagnóstico das fraturas nasais é realizado por meio de exames físico e de imagem. Durante o exame físico é necessário avaliar a presença de degraus ósseos e crepitações, presença de ferimento dermoabrasivo, edema, hematoma, desvio ou afundamento da pirâmide nasal, 
epistaxe, obstrução nasal por coágulo ou pelos fragmentos ósseos que se desprenderam da cavidade nasal, equimose periorbitaria bilateral, além do telecanto traumático.

Dentre os exames de imagem de maior aplicabilidade para diagnóstico desta modalidade de fratura estão a tomada radiográfica póstero anterior de Waters, perfil de face, bem como a tomografia computadorizada. ${ }^{1,5-7}$. Segundo Rohrich e Adams ${ }^{8}$, as fraturas nasais podem ser classificadas em cinco tipos: Tipo 1: fratura unilateral simples, Tipo 2: fratura bilateral, Tipo 3: fraturas cominutas, Tipo 4: lesão septal associada, e Tipo 5: fraturas naso-orbitoetmoidais.

O tratamento cirúrgico das fraturas nasais é controverso na literatura, sendo relatado por alguns autores que tratamentos de fraturas extensas envolvendo septo se restringem a redução aberta ${ }^{7,9}$, enquanto outros consideram a redução fechada o tratamento de escolha ${ }^{7,10}$.

O tratamento de redução fechada é realizado por meio de tamponamento nasal com gazes embebido em pomada antibiótica, inseridas em sentido posterior, a fim de proporcionar sustentação aos fragmentos fraturados. Podese utilizar também, externamente, um curativo de gesso para manutenção do contorno nasal, e também impedir o colapso dos fragmentos recém reduzidos ${ }^{11,12}$.

Eventualmente, outros achados podem se somar às fraturas OPN como alterações anatômicas prévias, as quais, mesmo não sendo resultantes do próprio trauma, podem ser corrigidas durante o tratamento. Assim, o objetivo do presente estudo consiste em descrever o tratamento de fratura OPN de paciente com desvio de septo original, o qual foi corrigido no mesmo momento cirúrgico.

\section{CASO CLÍNICO}

Paciente 38 anos, sexo masculino, melanoderma, vítima de agressão física foi admitido no Hospital Santa Casa de Misericórdia de Araçatuba, Araçatuba (SP)-Brasil, relatando dificuldade respiratória e dor na face. Ao exame físico notou-se rinoescoliose significativa no lado direito e impermeabilidade das vias aéreas, principalmente do lado direito (Figura 1). O exame de imagem por tomografia computadorizada revelou fratura OPN Tipo $4^{8}$ cominuta e desvio de septo nasal (Figuras 2 e 3 ).

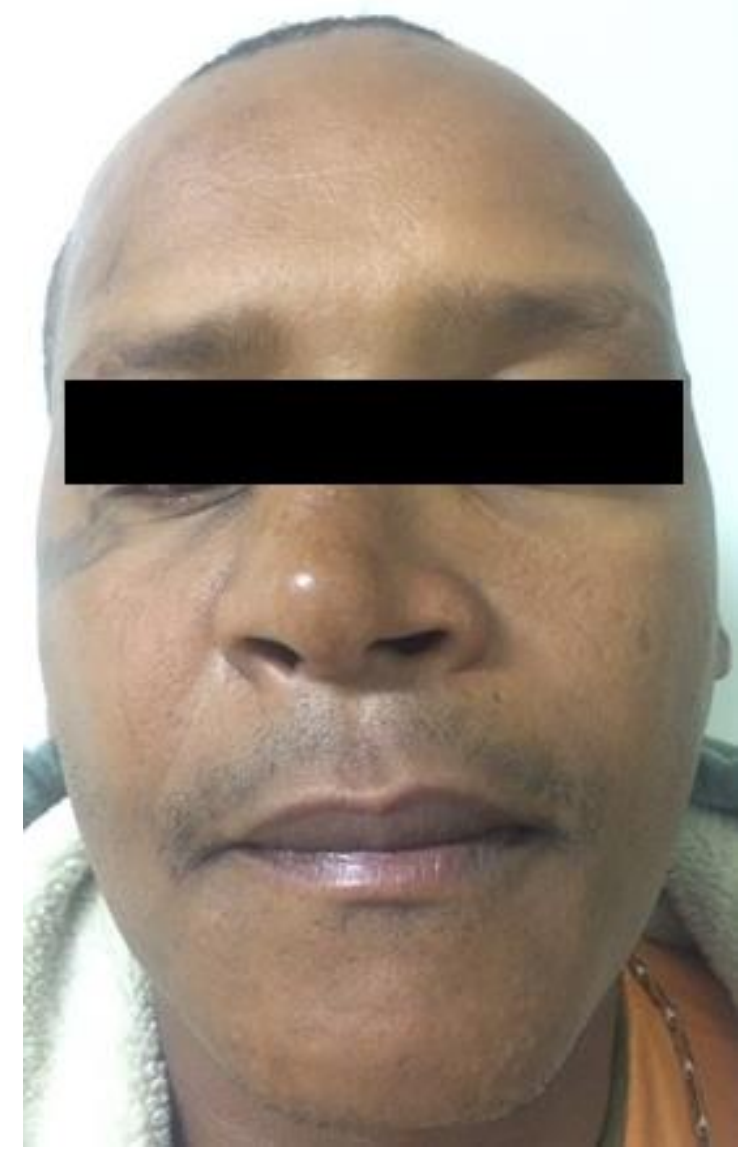

Figura 1: Exame clínico observando-se rinoescoliose à direita

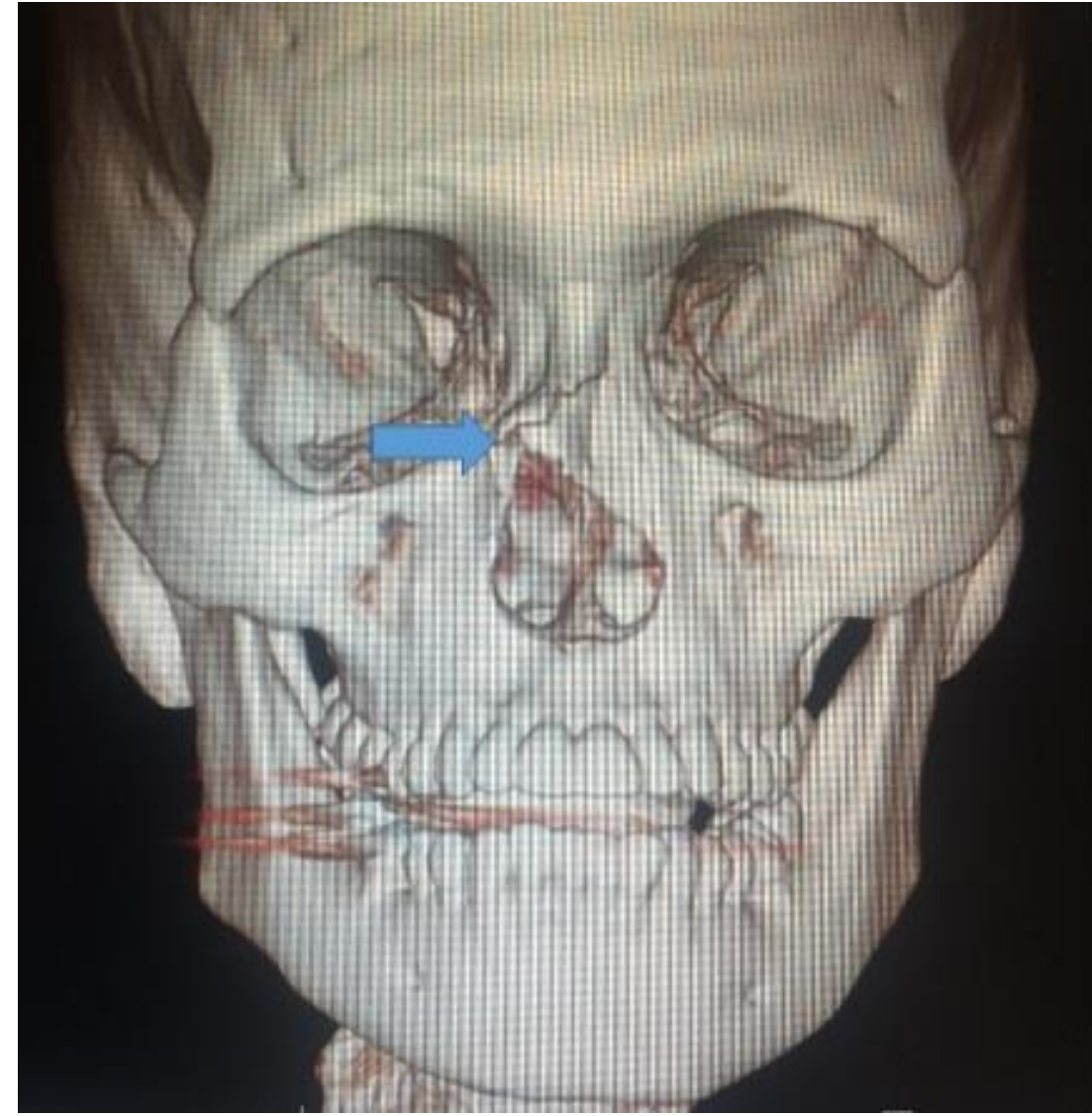

Figura 2: Exame de imagem com tomografia computadorizada em reconstrução 3D evidenciando fratura OPN com envolvimento de septo nasal.

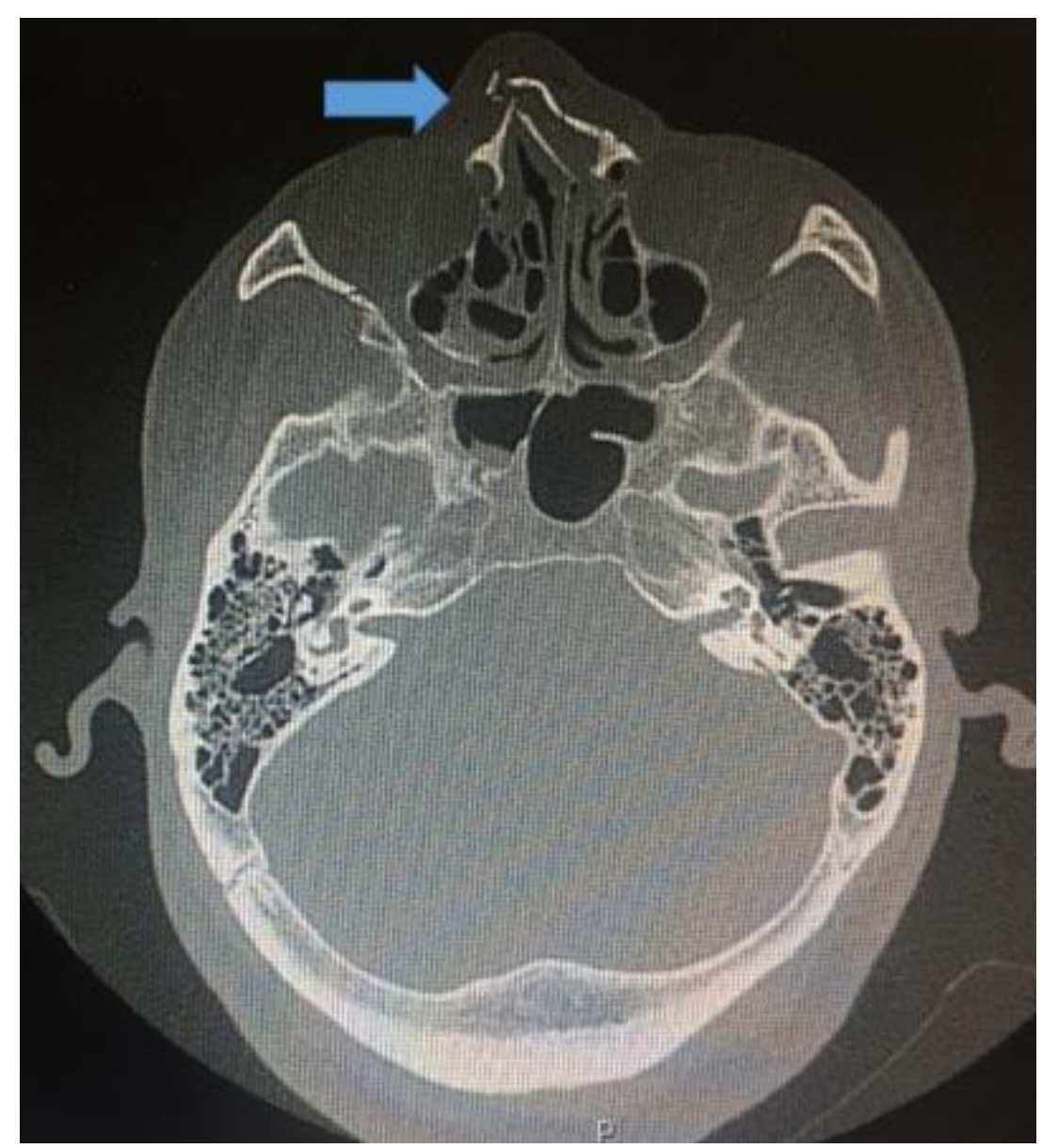

Figura 3: Corte axial evidenciando fratura OPN com envolvimento de septo nasal.

Pelas condições apresentadas no exame físico e de imagem foi proposto tratamento cirúrgico fechado sob anestesia geral e intubação orotraqueal. Realizou-se redução da fratura e do desvio de septo com o uso de fórceps de Asch seguido de tamponamento por 72 horas para sustentação e suporte, com colocação imediata de gesso externo para manutenção do suporte.

No retorno de sete dias pós-operatórios foi possível verificar resultados tais como vias áreas pérvias, com ausência de queixa álgica e estética satisfatória (Figuras 4 e 5). 


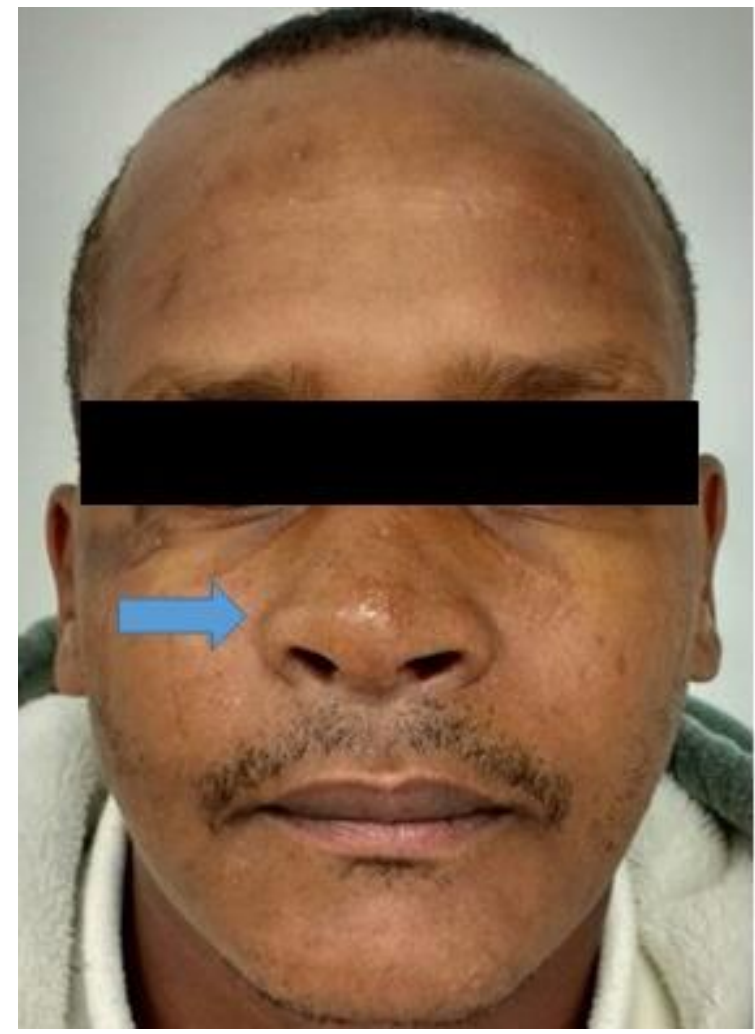

Figura 4: Pós-operatório de 10 dias.

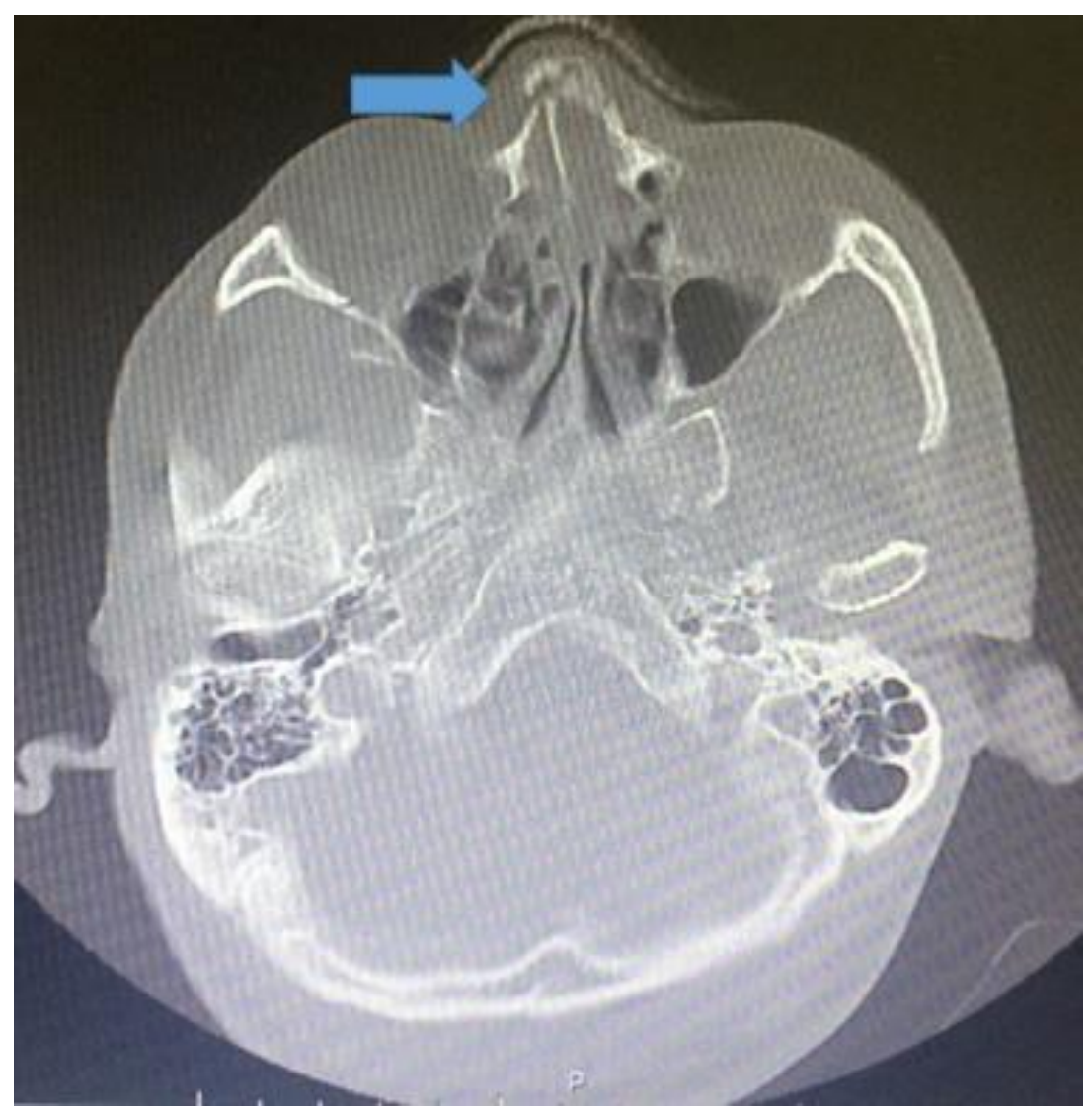

Figura 5: Tomografia computadorizada mostrando o correto posicionamento dos ossos próprios do nariz e do septo nasal.

\section{DISCUSSÃO}

A realização de redução de fraturas nasais pode ser executada por meio de redução aberta por meio de incisões e manipulação dos ossos nasais e do septo, ou pelo manejo da redução fechada com o uso de instrumentação e sem incisões. ${ }^{9,10}$

A escolha do tratamento de uma fratura OPN aberta ou fechada ainda é controversa. No presente caso, optou-se pela redução fechada na tentativa de minimizar o trauma e com isso estabilizar a fratura de OPN conforme a classificação de Rohrich e Adams ${ }^{8}$. A redução fechada está indicada para casos de fraturas unilaterais ou bilaterais de ossos nasais com envolvimento do septo ${ }^{13}$. Pelo fato de ter sido detectada fratura de septo decorrente do trauma, o tratamento cirúrgico envolveu o mesmo, o que norteou a escolha desta modalidade. Quanto à necessidade de se realizar anestesia geral, as intensas queixas álgicas do paciente reforçaram esta opção. A escolha entre anestesia local e geral é definida pelas experiências dos cirurgiões e características das fraturas apresentadas e do próprio paciente $^{3,14 .}$.

Uma gama de curativos pode ser utilizada no pósoperatório das fraturas de OPN, como o uso de esparadrapo, micropore e gesso, todos levando a um resultado final satisfatório $^{14,15}$. No caso clínico apresentado, elegeu-se o curativo de gesso a fim de manter intacta estrutura nasal reposicionada, sendo esse um dos métodos mais tradicionais dessa modalidade para manter o contorno nasal ${ }^{16}$.

\section{CONCLUSÃO}

Com base no tratamento proposto foi possível observar um prognóstico satisfatório no pós-operatório de 10 dias, com o restabelecimento do contorno nasal como também da função respiratória, sem sinais e sintomas flogísticos e leve edema, condição normal decorrente do procedimento cirúrgico realizado.

\section{REFERÊNCIAS}

1. Marzola C. Fundamentos de Cirurgia Buco Maxilo Facial. São Paulo: Big Forms; 2008.

2. Reis LF, Marzola C, Toledo Filho JL. Prevalência de fraturas faciais na região de Bauru, no período de janeiro de 1991 a dezembro de 1995. Rev Odonto Ciênc. 2001;16:34:231-40.

3. Dos Santos GM, Pires WR, Silva LF, De Deus CBD, Momesso GAC, Polo TOB. Tratamento cirúrgico de fratura severa de ossos próprios do nariz: relato de caso. Arch Health Invest. 2017; 6(4):189-91

4. Lockhart RD. Anatomia Humana. México, Editorial Interamericana, 1965.

5. Dingman RO, Nativig P. Cirurgia das fraturas faciais. Editora Santos, 1983.

6. Manganello-Souza LC, Luz JGC. Tratamento cirúrgico do trauma bucomaxilofacial. 3. ed. São Paulo: Roca; 2006

7. Monnazi MS, Oliveira HC, Passeri LA, Gabrielli MFR. Manejo das fraturas nasais com manutenção das vias aéreas superiores. Rev Cir Traumatol Buco-Maxilo-fac. 2010; 10(2):55-60.

8. Rohrich RJ, Adams WP Jr. Nasal fracture management: minimizing secondary nasal deformities. Plast Reconstr Surg. 2000; 106(2):266-73.

9. Fattahi T, Steinberg B, Fernandes R, Mohan M, Reitter E. Repair of nasal complex fractures and the need for secondary septo-rhinoplasty. J Oral Maxillofac Surg. 2006; 64(12):1785-9.

10. Bailey BJ. Head and Neck Surgery-Otolaryngology. Philadelphia: JB Lippincott Company; 2001. vol. 1, p.65-69.

11. Monnazi MS, Oliveira HC, Passeri LA, Gabrielli MFR. Manejo das fraturas nasais com manutenção das vias aéreas superiores. Rev Cir Traumatol Buco-Maxilo-Fac. 2010; 10(2):55-60.

12. Coto NP, Meira JBC, Dias RB. Fraturas nasais em esportes: sua ocorrência e importância. RSBO. 2010; 7(3):349-53.

13. Ziccardi VB, Braidy H. Management of nasal fractures. Oral Maxillofac Surg Clin North Am. 2009; 21(2):203-8.

14. Rajapakse Y, Courtney M, Bialostocki A, Duncan G, Morrissey G. Nasal Fractures: Astudy comparing local and general anaesthesia techniques. ANZ J Surg. 2003; 73(6):396-9. 10

15. de Almeida FS, Minarro LL, Pialarissi PR, Shirane E. Surgical Correction of the Saddle Nose: Case Report. Int. Arch. Otorhinolaryngol. 2009;13(4):450-4. 
16. Morgan DW, Deadman M. The thermoplastic splint (a simple effective alternative to plaster of Paris in the fixation of nasal fractures). J Laryngol Othol. 1988; 102(8):724.

\section{CONFLITO DE INTERESSES}

Os autores declaram não haver conflitos de interesse.

\section{AUTOR PARA CORRESPONDÊNCIA}

Gabriel Mulinari dos Santos gabriel_mulinari@hotmail.com

Submetido em 04/08/2017 Aceito em 04/09/2017 